\title{
Unusual presentation of Aspergillus aortitis after aortic valve surgery with massive hemoptysis
}

\author{
Lowell Leow, MBBS, MRCS (Edin), ${ }^{\mathrm{a}}$ Qi Qian, MD, MRCS (Edin), ${ }^{\mathrm{a}}$ Ka Lip Chew, MBBS, FRCPath, ${ }^{\mathrm{b}}$ and \\ Vitaly Sorokin, MD, PhD, MRCS (Edin), FRCS CTh (Edin), ${ }^{\mathrm{a}, \mathrm{c}}$ Singapore
}

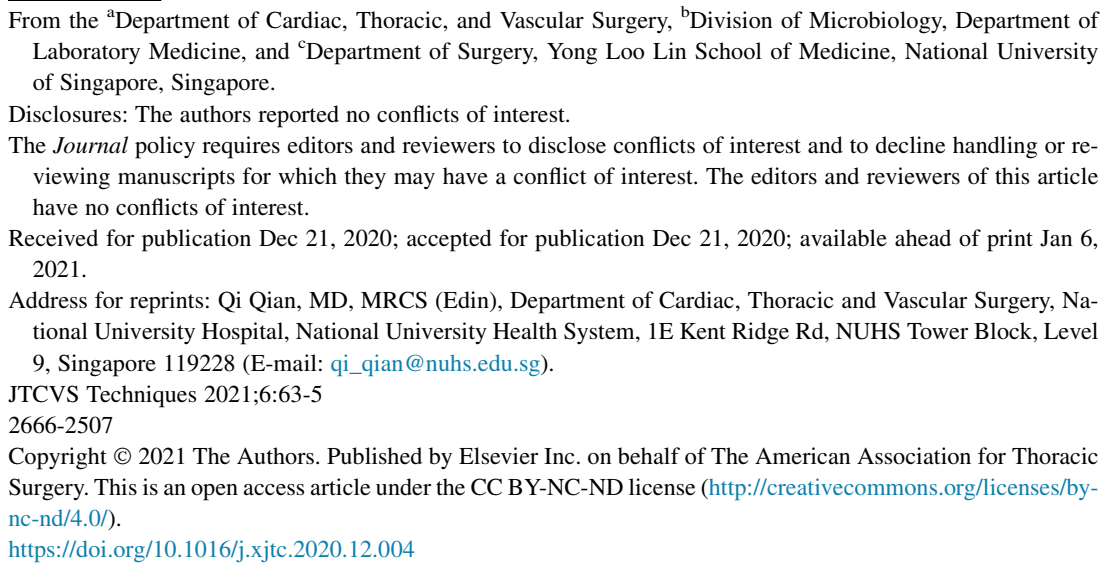

Invasive Aspergillus infection of the aorta after cardiac surgery is rare. ${ }^{1}$ Presentation varies greatly, and symptoms are nonspecific. ${ }^{2-4}$ Diagnosis is often delayed, and the prognosis is guarded despite intervention. ${ }^{1}$ We present a case of Aspergillus aortitis after aortic valve surgery presenting with massive hemoptysis due to ruptured aortic pseudoaneurysm.

\section{CASE PRESENTATION}

A 51-year-old man with a history of aortic valve and ventricular septal defect repair performed 23 years earlier and hyperlipidemia presented with New York Heart Association class III symptoms of aortic stenosis. The initial cardiac surgery was performed with patch repair of the ventricular septal defect and aortic valve repair with bovine pericardium. On follow-up, the mean transaortic gradient was $43 \mathrm{~mm} \mathrm{Hg}$, and the effective orifice area was $0.7 \mathrm{~cm}^{2}$. He underwent a redo sternotomy and aortic valve replacement (21-mm mechanical valve; St Jude Medical, St Paul, Minn). He recovered well and was discharged on postoperative day 8. Echocardiography showed a normal aortic valve prosthesis and ascending aorta.

At an outpatient follow-up 6 weeks later, the patient complained of cough with blood-stained secretions. He had no other respiratory symptoms and was afebrile and hemodynamically stable. His warfarin was titrated owing to an International Normalized Ratio (INR) of 3.0.

Despite the normalized INR, his hemoptysis persisted, and he presented again with decreased effort tolerance

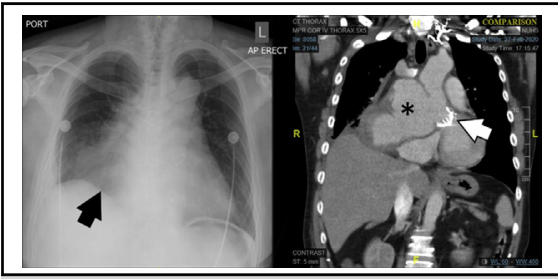

(Left) Chest X-ray showing potential lung infiltrate. (Right) Aspergillus fumigatus.

CENTRAL MESSAGE

We report an unusual presentation of Aspergillus aortitis in a patient after redo aortic valve surgery who was readmitted with lung consolidation and massive hemoptysis due to ruptured ascending aorta.

See Commentary on page 66.

and shortness of breath at rest with low-grade fever. A chest $\mathrm{X}$-ray showed right lower zone opacification with elevated right hemidiaphragm (Figure 1). He was diagnosed with right lower lobe pneumonia and treated with i.v. piperacillin/tazobactam. Echocardiography showed preserved ejection fraction with no pericardial effusion or valvular regurgitation. The next day, his hemoglobin dropped from the baseline of $8.5 \mathrm{~g} / \mathrm{dL}$ to $6.7 \mathrm{~g} / \mathrm{dL}$. His INR increased to 3.33 despite the withholding of warfarin. An urgent chest computed tomography scan revealed a $12.4 \times 10.3-\mathrm{cm}$ ascending aorta pseudoaneurysm compressing the right lung (Figure 2). There was moderate right pleural effusion with ground-glass changes in the middle and lower lobes. The patient was transferred to the intensive care unit immediately and warfarin reversed before undergoing emergency surgery.

Before intubation in the operating theatre, the patient had normal blood pressure with good peripheral saturation. $\mathrm{He}$ suddenly developed massive frank hemoptysis and pulseless electrical activity collapse right before intubation. Cardiopulmonary resuscitation with chest compression and intubation and cut-down femoral-femoral cardiopulmonary bypass (CPB) were initiated simultaneously. After 20 


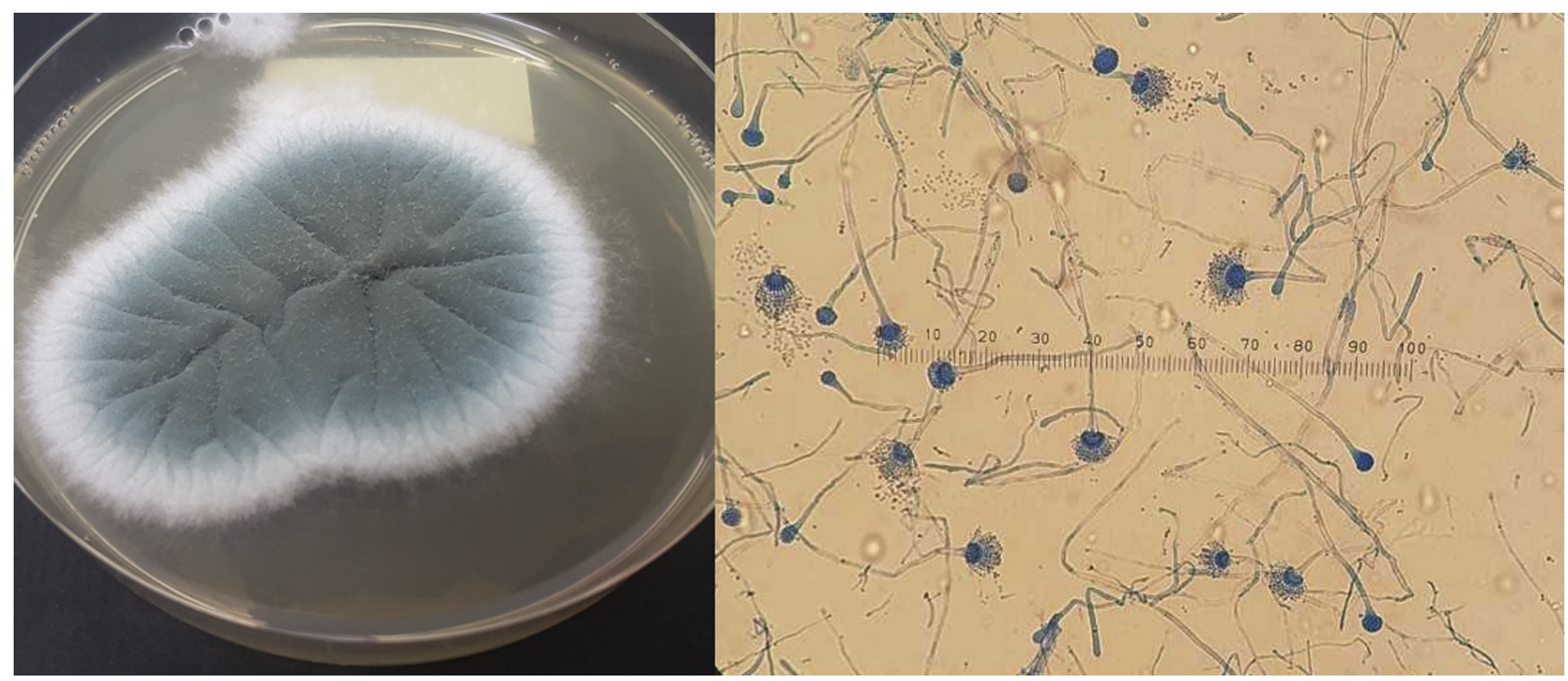

FIGURE 1. (Left) Macroscopic appearance of Aspergillus fumigatus; typical blue-green colony with suede-like appearance. (Right) Microscopic appearance, stained with lacto-phenol-cotton-blue at $200 \times$ magnification. Hyaline septate hyphae with typical sporulating dome-shaped vesicles are seen.

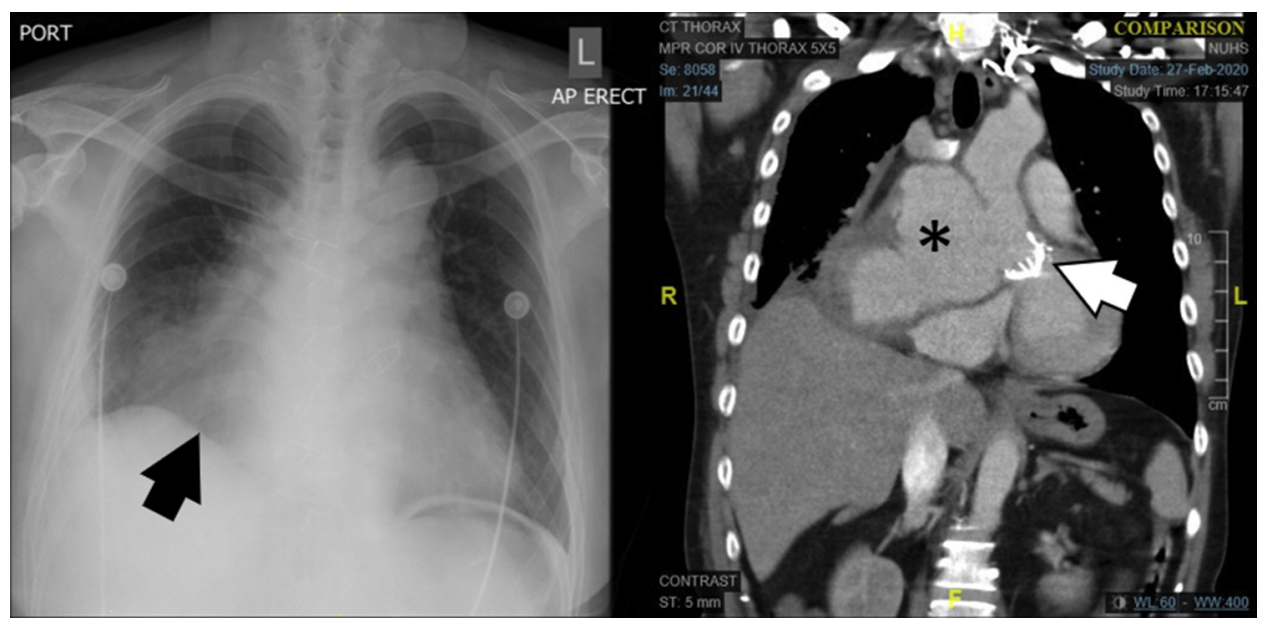

FIGURE 2. Chest X-ray showing right lower lobe consolidation (left $)$ and corresponding coronal thorax computed tomography scan showing the pseudoaneurysm (*) and mechanical aortic valve (right).

minutes of Cardiopulmonary resuscitation, CPB was established. The patient was cooled to $24^{\circ} \mathrm{C}$, and redo sternotomy was performed under circulatory arrest. The ascending aorta pseudoaneurysm was found to be ruptured above the aortotomy suture line for at least one-half of the aortic diameter. The aorta was cross-clamped proximal to the innominate artery, and CPB was reinstituted. Direct antegrade cardioplegia was administered. The mechanical valve was examined and found to be well sited.

The ascending aorta was replaced with a 26-mm straight Gelweave graft (Terumo, Sunrise, Fla). There was profuse bleeding from the right lung, caused by direct parenchymal erosion from the pressurized pseudoaneurysm. This was repaired primarily. The patient was weaned off CPB but required high inotropic support and ventilatory pressure; thus, peripheral venoarterial extracorporeal membrane oxygenation was instituted for circulatory and respiratory support.

Postoperatively, the patient's cardiorespiratory function recovered, and extracorporeal membrane oxygenation was discontinued on postoperative day 2. Unfortunately, his neurologic status showed severe hypoxic ischemic encephalopathy, likely related to his collapse and the downtime before reinstitution of $\mathrm{CPB}$.

Intraoperative culture of aortic wall tissue grew Aspergillus fumigatus on Sabouraud (Singapore) dextrose agar 
plates (at $30^{\circ} \mathrm{C}$ and $37^{\circ} \mathrm{C}$ ) after 1 day (Figure 1). Histology identified nonspecific inflammation and hemorrhage in the adventitia, with foreign body giant cell reaction to adjacent suture material.

\section{DISCUSSION}

Aspergillus aortitis is a rare but often fatal complication. ${ }^{1}$ Diagnosis is often delayed, and many patients are identified postmortem owing to the long incubation time for Aspergillus. ${ }^{4}$ In some instances, a diagnosis of pseudoaneurysm premortem is confirmed only on imaging. ${ }^{2}$ Despite antifungal treatment and excision of infected tissue, many patients still die after experiencing initial recovery. ${ }^{4,5}$ In the present case, Aspergillus aortitis presented with symptoms suggestive of pneumonia. Low-grade fever could be overlooked by the patient and was not reported at follow-up. The ascending aorta pseudoaneurysm was revealed only on computed tomography. Pericardial effusion or tamponade was prevented by scar tissue owing to his previous surgeries. This limited the pseudoaneurysm and explained why no collections were seen on echocardiography. Under high aortic pressures, the pseudoaneurysm had eroded into the lung and caused massive hemoptysis.

In conclusion, invasive Aspergillus infection of the aorta is a rare and aggressive complication with high mortality. Early identification is the only way to improve patient survival. A high suspicion for Aspergillus infection is required after aortic valve surgery, especially if unusual symptoms present.

\section{References}

1. Sanchez-Recalde A, Mate I, Merino JL, Simon RS, Sobrino JA. Aspergillus aortitis after cardiac surgery. J Am Coll Cardiol. 2003;41:152-6.

2. Ronco F, Simsir S, Czer L, Luo H, Siegel RJ. Incidental finding by twodimensional echocardiography of a mycotic pseudoaneurysm of the ascending aorta after orthotopic heart transplantation. J Am Soc Echocardiogr. 2010;23: 580.e1-3.

3. Sharifulin R, Bogachev-Prokophiev A, Varnek Y, Klimko N, Malakhova O, Volkov A, et al. Successful treatment of an aortotomy site Aspergillus infection. Ann Thorac Surg. 2019;108:e253-5.

4. Hanvesakul R, Deshpande R, Carr CS, Akbar N. Aspergillus aortitis: a cause for aortic perforation in a patient following combined aortic valve surgery and liver transplantation. Interact Cardiovasc Thorac Surg. 2004;3:544-6.

5. Clarke NS, Sengupta A, Miller A, Jessen ME, Murthy RA. Aspergillus aortitis and aortic valve endocarditis after coronary surgery. J Card Surg. 2019;34:871-4. 